\title{
Insights from the zero-angular-momentum wave in single and double ionization of He by fast electrons
}

\author{
M. J. Ambrosio, ${ }^{1,}{ }^{*}$ G. Gasaneo, ${ }^{1}$ and F. D. Colavecchia ${ }^{2}$ \\ ${ }^{1}$ Departamento de Física, Universidad Nacional del Sur and Consejo Nacional de Investigaciones Científicas y Técnicas, 8000 Bahía Blanca, \\ Buenos Aires, Argentina \\ ${ }^{2}$ División Física Atómica, Molecular y Óptica, Centro Atómico Bariloche and Consejo Nacional de Investigaciones Científicas y Técnicas, \\ 8400 S. C. de Bariloche, Río Negro, Argentina \\ (Received 17 October 2013; published 30 January 2014)
}

\begin{abstract}
In a recent contribution our group proposed a perturbative approach to deal with the open problem of double ionization of helium by electron impact. The goal of this work is to delve into the physics of the scattering function, and provide evidence of the nature of the single-ionization channels present in the three-body wave function. We show that the fast oscillating parts of the wave function are indeed the single-ionization channels, which appear coupled with the double continuum ones. We found a particular type of basis set within the Generalized Sturmian method that is better suited to isolate single from double-ionization channels.
\end{abstract}

DOI: 10.1103/PhysRevA.89.012713

PACS number(s): 34.80.Bm, 34.50.Fa, 31.15.A-

\section{INTRODUCTION}

The double ionization of atoms by electron impact, even in the simplest case of helium targets, is still an open problem in atomic physics [1]. Even the most sophisticated ab initio theories are unable to describe absolute experimental data without the need for a rescaling. The differences are specially important in the high-energy regime, where experimental absolute fivefold differential cross sections are available; see for example, Lahmam-Bennani et al. and Taouil et al. [2,3].

In the past two decades computer performance has seen great progress, which enabled physicists to develop more and more sophisticated numerical approaches to solve problems with a greater degree of accuracy. Examples of these methods are the time independent methods, such as the exterior complex scaling (ECS) [4], the convergent close coupling (CCC) [5,6], and $J$ matrix [7-9], among others.

Part of the complexity of three-body Coulomb problems lies in the fact that asymptotic conditions are difficult to include in the models, and each method approaches this problem in a different way.

The ECS methodology takes advantage of a rotation of the radial coordinates to the complex plane. This allows us to include the correct asymptotic behavior without enforcing it formally.

The CCC scheme distinguishes each of the ejected electrons. One electron is represented in terms of basis states obtained from an $\mathcal{L}^{2}$ diagonalization of the target Hamiltonian, which includes pseudostates for the continuum part of the spectrum. The other one is described by continuum Coulomb wave functions. The three-body wave function is not computed explicitly, but the $T$-matrix elements are obtained instead. Three-body outgoing behavior is set by using the Green operator in a simplified Lippmann-Schwinger equation for the $T$ matrix. Thus, the asymptotic behavior is explicitly enforced in this methodology.

The $J$-matrix model takes a similar path to CCC [8-10]. Outgoing-type behavior is explicitly enforced for one of the

\footnotetext{
*Corresponding author: marcelo.ambrosio@uns.edu.ar
}

electrons, while standing waves are selected for the other one. The method separates the three-body wave function solution in the internal and the external part. The internal wave function is expanded in terms of three-body Hamiltonian eigenfunctions obtained trough a $\mathcal{L}^{2}$ diagonalization. The external one is represented in terms of Coulomb Sturmian functions with outgoing asymptotic behavior. The coefficients of these expansions are obtained by solving a linear system of equations. Thus, the explicit form of the asymptotic conditions is used.

Time-dependent models, such as the time-dependent close coupling $[11,12]$, the time-dependent exterior complex scaling (TDECS) [13] or a wave-packet evolution [14], use completely different types of asymptotic conditions and avoid the difficulties of the time-independent methods.

One of the aims of this contribution is to show that the generalized Sturmian functions (GSFs) theory impose the desired asymptotic behavior, for any (single or double) ionization channel. This is done by imposing outgoing behavior on each of the radial coordinates of two sets of spherical coordinates, one for each ejected electron. Within the GSF method, outgoing asymptotic conditions are explicitly enforced on each coordinate, which in turn enforces the correct three-body behavior.

Let us now recall the history of the GSF method. The group begun implementing GSF with the work of Frapiccini et al. [15], where the main aspects of the methodology were introduced. References [16,17] contain a good summary on the mathematical properties of Sturmian basis sets.

The group has also presented some applications to two$[16,18]$ and three-body bound-state problems $[17,19,20]$. Sturmian functions for bound states have been shown to be very efficient, and one can obtain highly accurate free $[17,19,20]$ and confined [21] helium eigenvalues among many other systems [22].

Continuum GSFs have also been implemented to solve three-body scattering problems in Refs. [23,24]. In all these examples, the GSF method has proven to be efficient and accurate. For a complete review see Ref. [22].

The aim of the present contribution is to analyze the information contained in the wave function of an $S$-wave 
model for double ionization of helium by fast electrons, with the GSF method. The present work can be viewed as a continuation of that started in [25], but the attention now is focused on the physics contained in the scattering wave function. Indeed, we will show that the wave function contains both single and double-ionization channels. We discuss a particular type of basis set that is more adequate to separate contributions from these different ionization channels that are embedded in the solution.

The outline of this paper is as follows. In Sec. II we summarize the basics of the generalized Sturmian functions and their mathematical properties, and present the relevant details of two particular choices of basis sets. In Sec. III, we present the theoretical framework for fast electron double ionization of helium. In Sec. IV we compare the three-body wave function of the problem obtained with both bases presented before. In Sec. V, we turn our attention to the structures arising at the edges of the radial domains for both target electrons, and show that they are related directly to single-ionization channels. We also present the pros and cons of each of the basis sets used. Finally, we summarize our results in Sec. VI. Atomic units $(\hbar=e=1)$ are used throughout.

\section{GENERALIZED STURMIAN FUNCTIONS}

\section{A. Basic theory}

Let us start with a brief description of the mathematical properties of the generalized Sturmian functions $S_{n l}(r)$. They are the solutions of a radial Schrödinger equation,

$$
\left[\mathcal{T}_{l}+\mathcal{U}(r)-E_{s}\right] S_{n l}(r)=-\beta_{n l} \mathcal{V}(r) S_{n l}(r),
$$

where $\mathcal{T}_{l}=-\frac{1}{2} \frac{d^{2}}{d r^{2}}+\frac{l(l+1)}{2 r^{2}}$ is the radial kinetic-energy operator. The main feature of the Sturmians functions is that they constitute a complete set, where all of them share the same asymptotic behavior. The auxiliary potential $\mathcal{U}(r)$ can be chosen as a suitable representation of the physics of a particular problem. It may even have a long-range Coulomb tail. The generating potential $\mathcal{V}(r)$ is an arbitrary short-range interaction. All the Sturmian elements have the same energy, which shapes their asymptotic behavior, together with the potential $\mathcal{U}(r)$. Sturmian functions can be chosen to have pure incoming or outgoing flux, which results in complex eigenvalues $\beta_{n l}$, since probability has to be created or destroyed to have those conditions at the boundary. We chose outgoing wave asymptotic conditions throughout this paper:

$$
S_{n l}(r) \rightarrow H_{l}^{(+)}\left(Z, E_{s}, r\right),
$$

where $H_{l}^{(+)}\left(Z, E_{s}, r\right)$ is the pure outgoing Coulomb function, with charge $Z$, energy $E_{s}$, and angular momentum $l$ [26].

Generalized Sturmian functions satisfy closure and orthogonality relations because they are solutions of a SturmLiouville problem; see for example [27,28]. It is important to note that in our methodology no complex conjugation is used in the orthogonality or closure identities.

\section{B. Basis set types}

There are two well-known generating potentials $\mathcal{V}(r)$ that have proven to be useful for the analysis of the doubleionization of atoms by electron impact. These are the square- well (SW) and the Yukawa potential. The three-body solutions that are obtained with them are equivalent, although the basis functions they produce differ considerably. In the three-body context, the SW basis was used in [23], and both types were used in [24], where they were generated with two different methodologies $[15,20]$.

\section{Generalized Sturmian functions generated by a square-well (SW) potential}

The square-well potential is constant and negative for a radial interval $\left(0, r_{\max }\right)$ :

$$
\mathcal{V}(r)=\left\{\begin{array}{l}
-1 \text { for } r \leqslant r_{\max }, \\
0 \text { otherwise }
\end{array}\right.
$$

Since Sturmians are orthogonal with respect to the generating potential [29] the orthogonality relation is similar to that of energy eigenfunctions in the SW case. However, those energy eigenfunctions belong to a continuum spectrum and have no net flux. This feature makes them less attractive for scattering problems where the asymptotic behavior implies positive or negative flux.
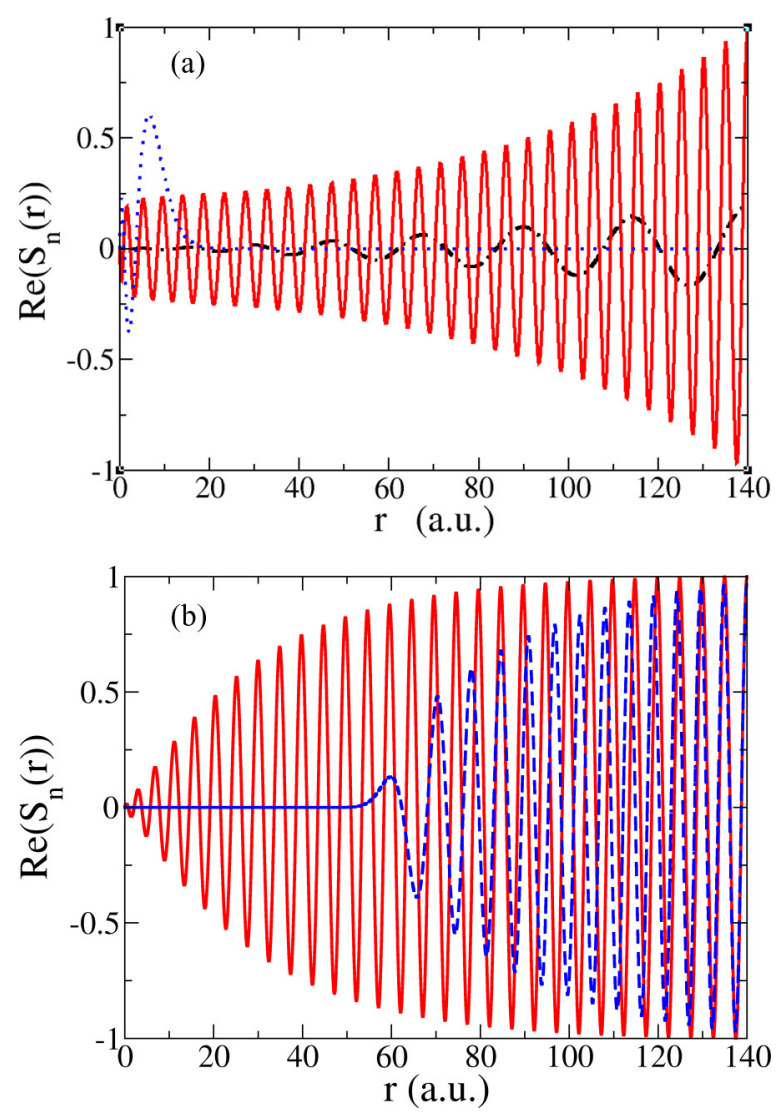

FIG. 1. (Color online) Real part of Sturmian functions computed with a square-well (a) or Yukawa potential (b). The three types of SW Sturmians: one resembling auxiliary potential bound eigenstates (dotted line), one with continuumlike behavior with repulsive (i.e., negative) real part eigenvalue $\beta_{n 0}$ (dashed line), and one with positive real part (i.e., attractive) eigenvalue (solid line). Yukawa Sturmians (b) present only attractive (solid line) or repulsive (dashed line) behavior. 
In Fig. 1(a) we show the real parts of three basis elements computed with a SW as generating potential. Most of the SW Sturmian functions gradually gain amplitude as the radial variable grows, reflecting the fact that probability is being created by the complex valued $\beta_{n l} \mathcal{V}(r)$. However, a small subset of the basis is GSFs that resemble bound states of the auxiliary potential. Their eigenvalues satisfy the condition $\mathcal{R} e\left(\beta_{n l}\right)<0$. In the present work the radial grid extends up to 140 a.u., and using the helium nuclear potential $\mathcal{U}(r)=\frac{-2}{r}$, nine functions of the basis are boundlike states.

\section{Generalized Sturmian functions generated by a Yukawa potential}

This is the screened version of the, long ranged, Coulomb interaction:

$$
\mathcal{V}(r)=-\frac{e^{-\alpha r}}{r} .
$$

The introduction of this generating potential helps to concentrate the basis density in a region closer to the origin. The $\alpha>0$ parameter regulates the degree of this feature. An illustration of these states is plotted in Fig. 1(b), where we show the real parts of two basis GSF elements. Continuum Yukawa basis functions, as their SW counterparts, also gain amplitude as $r$ grows, however they do so more abruptly due to the shape of their generating potential. We have shown that negative energy Yukawa-type basis functions are a very efficient basis set for calculation of helium bound states [19,20].

\section{FAST ELECTRON REGIME MODEL}

Let us consider the description of the ionization of neutral atoms by electron impact. In a typical experiment a beam of electrons is directed to a low-density cloud of neutral atoms forming a jet stream set up inside of a collision chamber. The electron projectiles are accelerated to the final energy far from the collision region. Here we will consider the case where these projectiles have very high energy. We will consider an $\mathrm{He}$ atom as a target. After the collision the projectile electron is again far away from the target, transferring some energy to it. Let us suppose that $E_{a}$ is the energy of the He subsystem, above the double-ionization threshold. There are two possible outcomes of the collision in this regime: single and double ionization. For single ionization, one of the target electrons is ejected into the continuum, while the second one remains in a (possibly excited) state, bound to the nucleus. For double ionization, both electrons are emitted into a double continuum. In that state they share their energies $E_{2}$ and $E_{3}$ such that $E_{a}=E_{2}+E_{3}$. All these states result from the same collision process, and therefore all of them should be included in the full solution of the Schrödinger equations describing the system:

$$
\begin{gathered}
\left(-\frac{1}{2} \nabla_{1}^{2}-\frac{1}{2} \nabla_{2}^{2}-\frac{1}{2} \nabla_{3}^{2}-\frac{Z}{r_{1}}-\frac{Z}{r_{2}}-\frac{Z}{r_{3}}\right. \\
\left.+\frac{1}{r_{12}}+\frac{1}{r_{13}}+\frac{1}{r_{23}}-E\right) \Psi\left(\mathbf{r}_{1}, \mathbf{r}_{2}, \mathbf{r}_{3}\right)=0 .
\end{gathered}
$$

Here particle 1 is designed to be the electronic projectile; particles 2 and 3 are the target electrons, while $Z$ is the nuclear charge. To separate the initial state from the final one, we write the wave function in (5) as

$$
\Psi\left(\mathbf{r}_{1}, \mathbf{r}_{2}, \mathbf{r}_{3}\right)=\frac{1}{(2 \pi)^{3 / 2}} e^{i \mathbf{k}_{i} \cdot \mathbf{r}_{1}} \Phi_{i}\left(\mathbf{r}_{2}, \mathbf{r}_{3}\right)+\Psi_{s c}^{+}\left(\mathbf{r}_{1}, \mathbf{r}_{2}, \mathbf{r}_{3}\right) .
$$

Here we assumed a high-energy regime for the projectile, which is described by plane wave with momentum $\mathbf{k}_{i}$. The initial ground state of the target is defined by $\Phi_{i}\left(\mathbf{r}_{2}, \mathbf{r}_{3}\right)$. Replacing $\Psi\left(\mathbf{r}_{1}, \mathbf{r}_{2}, \mathbf{r}_{3}\right)$ as given in (6) leads to the equation satisfied by the scattering function $\Psi_{s c}^{+}\left(\mathbf{r}_{1}, \mathbf{r}_{2}, \mathbf{r}_{3}\right)$ :

$$
\begin{aligned}
& \left(-\frac{1}{2} \nabla_{1}^{2}+h_{H e}-\frac{Z}{r_{1}}+\frac{1}{r_{12}}+\frac{1}{r_{13}}-E\right) \Psi_{s c}^{+}\left(\mathbf{r}_{1}, \mathbf{r}_{2}, \mathbf{r}_{3}\right) \\
& =-\left(-\frac{Z}{r_{1}}+\frac{1}{r_{12}}+\frac{1}{r_{13}}\right) \frac{e^{i \mathbf{k}_{i} \cdot \mathbf{r}_{1}}}{(2 \pi)^{3 / 2}} \Phi_{i}\left(\mathbf{r}_{2}, \mathbf{r}_{3}\right)
\end{aligned}
$$

where $h_{H e}$ is the three-body helium Hamiltonian

$$
h_{H e}=\left(-\frac{1}{2} \nabla_{2}^{2}-\frac{1}{2} \nabla_{3}^{2}-\frac{Z}{r_{2}}-\frac{Z}{r_{3}}+\frac{1}{r_{23}}\right) .
$$

After the collision takes place, the energy of the scattered projectile is still very high. Therefore, we can use a plane-wave representation for $\Psi_{s c}^{+}\left(\mathbf{r}_{1}, \mathbf{r}_{2}, \mathbf{r}_{3}\right)$,

$$
\Psi_{s c}^{+}\left(\mathbf{r}_{1}, \mathbf{r}_{2}, \mathbf{r}_{3}\right)=\int d \mathbf{k} \frac{e^{i \mathbf{k} \cdot \mathbf{r}_{1}}}{(2 \pi)^{3 / 2}} \Phi_{s c}^{+}\left(\mathbf{r}_{2}, \mathbf{r}_{3}\right)
$$

The projectile final momentum is $\mathbf{k}_{f}$. Thus, we can replace the proposal (9) into (7) and project with a plane wave with the final momentum of the projectile,

$$
\begin{aligned}
& \left(h_{H e}-E_{a}\right) \Phi_{s c}^{+}\left(\mathbf{r}_{2}, \mathbf{r}_{3}\right) \\
& \quad+4 \pi \int d \mathbf{k} \frac{\left(-Z+e^{i \mathbf{p} \cdot \mathbf{r}_{2}}+e^{i \mathbf{p} \cdot \mathbf{r}_{3}}\right)}{p^{2}} \Phi_{s c}^{+}\left(\mathbf{p}, \mathbf{r}_{2}, \mathbf{r}_{3}\right) \\
& =-\frac{4 \pi}{q^{2}}\left(-Z+e^{i \mathbf{q} \cdot \mathbf{r}_{2}}+e^{i \mathbf{q} \cdot \mathbf{r}_{3}}\right) \Phi_{i}\left(\mathbf{r}_{2}, \mathbf{r}_{3}\right) .
\end{aligned}
$$

Here $\mathbf{q}=\mathbf{k}_{i}-\mathbf{k}_{f}$ is the momentum transferred to the target by the projectile. In the same way, we define $\mathbf{p}=\mathbf{k}-\mathbf{k}_{f}$.

Under the situation of a high-energy projectile and very small energy transfer to the target, the term with the integral on the left-hand side of (10) can be neglected, and we end up with the equation

$$
\begin{aligned}
\left(h_{H e}-E_{a}\right) \Phi_{s c}^{+}\left(\mathbf{r}_{2}, \mathbf{r}_{3}\right)= & -\frac{1}{(2 \pi)^{3}} \frac{4 \pi}{q^{2}}\left(-Z+e^{i \mathbf{q} \cdot \mathbf{r}_{2}}+e^{i \mathbf{q} \cdot \mathbf{r}_{3}}\right) \\
& \times \Phi_{i}\left(\mathbf{r}_{2}, \mathbf{r}_{3}\right),
\end{aligned}
$$

which is equivalent to the first Born approximation [25].

At this point, it is necessary to detail the asymptotic behavior of $\Phi_{s c}^{+}\left(\mathbf{r}_{2}, \mathbf{r}_{3}\right)$. As we mentioned before, $\Phi_{s c}^{+}\left(\mathbf{r}_{2}, \mathbf{r}_{3}\right)$ should have different asymptotes for each ionization channel. For example, when one of the electrons is close to the nucleus in a bound state, while the other one in the continuum is far away from them, the wave function should have the asymptotic form

$\Phi_{s c, 2}^{+}\left(\mathbf{r}_{2}, \mathbf{r}_{3}\right) \underset{r_{2} \rightarrow \infty}{\rightarrow} \frac{1}{2 \pi} \sum_{n} F_{n}\left(k_{n} \hat{\mathbf{r}}_{2}, \mathbf{q}\right) \frac{e^{i k_{n} r_{2}-i \eta_{2, n} \ln \left(2 k_{n} r_{2}\right)}}{r_{2}} \phi_{n}\left(\mathbf{r}_{3}\right)$.

These are the asymptotic conditions in the $\Omega_{i}$ regions (with $i=2,3$ ), named after the work of Alt and Mukhamedzhanov 
[30]. Here $\eta_{2, n}$ is the Sommerfeld parameter $\eta_{2, n}=Z / k_{n}$. The sum in (12) represents the situation where one of the electrons remains bound in the state $\phi_{n}\left(\mathbf{r}_{3}\right)$ and the other ends up in the continuum; $F_{n}\left(k_{n} \hat{\mathbf{r}}_{1}, \mathbf{q}\right)$ is the single-ionization transition amplitude. The symmetric case is represented by the wave function $\Phi_{s c, 3}^{+}\left(\mathbf{r}_{2}, \mathbf{r}_{3}\right)$. The summation in Eq. (12) runs only over bound states.

When both electrons are in the continuum, the corresponding asymptotic region is named $\Omega_{0}$, and all three particles are far from each other [30]. In such case no contributions coming from bound states are expected in the asymptotic three-body scattering wave function since all interparticle distances are large. The hyperspherical wave is known to be the correct solution [31-33]:

$$
\Phi_{s c, c}^{+}\left(\mathbf{r}_{2}, \mathbf{r}_{3}\right) \underset{\rho \rightarrow \infty}{\rightarrow}(2 \pi i)^{(1 / 2)} T_{\tilde{\mathbf{k}}_{2}, \tilde{\mathbf{k}}_{3}} \frac{\kappa^{3 / 2}}{\rho^{5 / 2}} e^{i \kappa \rho+i \lambda_{0} \ln (2 \kappa \rho)+i \pi / 4},
$$

where $\lambda_{0}$ is a Coulomb parameter and $T_{\tilde{\mathbf{k}}_{2}, \tilde{\mathbf{k}}_{3}}=T\left(\frac{\kappa}{\rho} \mathbf{r}_{2}, \frac{\kappa}{\rho} \mathbf{r}_{3}\right)$ is the double-ionization transition amplitude. The coordinatedependent momenta $\tilde{\mathbf{k}}_{j}(j=2,3)$ were defined originally by Alt and Mukhamedzhanov [30], while $\kappa=\sqrt{k_{2}^{2}+k_{3}^{2}}$ is the hypermomentum of the particles.

When the collision process is studied and the Schrödinger equation is solved numerically, all the channels, represented by the asymptotic wave functions $\Phi_{s c, 2}^{+}\left(\mathbf{r}_{2}, \mathbf{r}_{3}\right), \Phi_{s c, 3}^{+}\left(\mathbf{r}_{2}, \mathbf{r}_{3}\right)$ and $\Phi_{s c, c}^{+}\left(\mathbf{r}_{2}, \mathbf{r}_{3}\right)$, are coupled and included simultaneously into the solution. In other words, the full solution of (10) should have the following general form at large distances [34]:

$$
\Phi_{s c}^{+}\left(\mathbf{r}_{2}, \mathbf{r}_{3}\right) \underset{\rho \rightarrow \infty}{\rightarrow} \Phi_{s c, 2}^{+}\left(\mathbf{r}_{2}, \mathbf{r}_{3}\right)+\Phi_{s c, 3}^{+}\left(\mathbf{r}_{2}, \mathbf{r}_{3}\right)+\Psi_{s c, c}^{+}\left(\mathbf{r}_{2}, \mathbf{r}_{3}\right) .
$$

To analyze the ability of the generalized Sturmian methodology to generate a solution containing all the channels, we consider the simpler case of an $S$-wave model [25]:

$$
\begin{gathered}
{\left[-\frac{1}{2 r_{2}^{2}} \frac{\partial}{\partial r_{2}}\left(r_{2}^{2} \frac{\partial}{\partial r_{2}}\right)-\frac{1}{2 r_{3}^{2}} \frac{\partial}{\partial r_{3}}\left(r_{3}^{2} \frac{\partial}{\partial r_{3}}\right)-\frac{Z}{r_{2}}\right.} \\
\left.-\frac{Z}{r_{3}}+\frac{1}{r_{>}}-E_{a}\right] \phi_{s c}^{+}\left(r_{2}, r_{3}\right)=\mathcal{F}\left(r_{2}, r_{3}\right),
\end{gathered}
$$

where the right-hand side (RHS) is given by

$$
\mathcal{F}\left(r_{2}, r_{3}\right)=-\frac{1}{(2 \pi)^{3}} \frac{4 \pi}{q^{2}}\left[-Z+j_{0}\left(q r_{2}\right)+j_{0}\left(q r_{3}\right)\right] \phi^{(0)}\left(r_{2}, r_{3}\right) .
$$

Here $j_{0}(x)$ is the zero-order spherical Bessel function. The initial state $\phi^{(0)}\left(r_{2}, r_{3}\right)$ in (16) is the ground-state solution of the $S$-wave helium equation [25].

The solution of (15), $\phi_{s c}^{+}\left(r_{2}, r_{3}\right)$, is expanded in terms of the uncorrelated spherical generalized Sturmian basis set

$$
\phi_{s c}^{+}\left(r_{2}, r_{3}\right)=\sum_{n_{a} n_{b}} a_{n_{a} n_{b}} \Xi_{n_{a}, n_{b}}\left(r_{2}, r_{3}\right),
$$

where

$$
\begin{aligned}
& \Xi_{n_{a}, n_{b}}\left(r_{2}, r_{3}\right) \\
& \quad=\frac{1}{\sqrt{2}}\left[\frac{S_{n_{a}}^{+}\left(r_{2}\right)}{r_{2}} \frac{S_{n_{b}}^{+}\left(r_{3}\right)}{r_{3}}+(-1)^{S} \frac{S_{n_{a}}^{+}\left(r_{3}\right)}{r_{3}} \frac{S_{n_{b}}^{+}\left(r_{2}\right)}{r_{2}}\right] .
\end{aligned}
$$

and $S_{n}^{+}(r)$ is a GSF with outgoing ( + ) behavior and $l=0$.

Replacing expansion (17) in (15), and projecting into basis element (18) yields the following system of equations:

$$
\left[\mathbf{H}_{\mathbf{T P}}-\left(E_{a}-E_{1}-E_{2}\right) \mathbf{O}\right] \mathbf{a}=\mathbf{F},
$$

where $\mathbf{H}_{\mathbf{T P}}$ is the matrix representation of the operators in (15), separating the overlapping term, whose elements $\operatorname{are} \mathbf{O}_{n_{a}, n_{a}}^{n_{a}^{\prime}, n_{b}^{\prime}}=$ $\left\langle\boldsymbol{\Xi}_{n_{a}^{\prime}, n_{b}^{\prime}} \mid \boldsymbol{\Xi}_{n_{a}, n_{b}}\right\rangle$. Similarly, the $\mathbf{F}$ vector is the projection of the RHS of Eq. (16) onto $\Xi_{n_{a}, n_{b}}\left(r_{2}, r_{3}\right)$.

\section{THREE-BODY PROBLEM SOLUTION}

In the fast projectile regime we are considering, the original four-body problem reduces to a three-body one, where the wave function of the two target electrons is unknown. These electrons can, after the collision has taken place, be both ionized, or one of them ionized and the other one remains bound in a $\mathrm{He}^{+}$ionic state. The Sturmian basis set has an energy $E_{s t}=E$, i.e., set to the total energy available for both target electrons. We have to point out, however, that $E_{s t}$ is in principle an arbitrary mathematical tool, and can be chosen, for example, to improve the convergence properties of the method. The boundary of the box was set at $r_{2}=r_{3}=140$ a.u. Each of the two-body basis sets verifies (1).

The $S$-wave helium bound state is generated with a negative energy Yukawa basis set. For negative energies and bound states, Yukawa potentials were found to be the best alternative within our GSF methodology [19,20].

In Fig. 2 we show the result of our calculations obtained with GSF using each of the generating potentials discussed in the precedent section: in panel (a) we show the wave function obtained with a SW potential, while the result of the calculation with a Yukawa potential is plotted in panel (b). While for most of the $\left(r_{2}, r_{3}\right)$ domain both functions yield similar results, there are some differences near the edges $r_{2}=0$ or $r_{3}=0$. One can see the hyperspherical wave front of Eq. (13) on a wide region of the coordinates far from the borders, and is especially clear for larger values of $r_{2}$ and $r_{3}$. Near the borders the frequency of oscillations of the wave function does not match the one of the hyperspherical front (see Fig. 3). Besides, taller peaks are present there, which are more clearly visible in Fig. 2(a). These results were obtained for the situation where the energy given by the projectile to the target exceeds the double-ionization threshold.

We observe that the solution obtained with the SW basis can represent the wave function near the edges to larger distances. In the double continuum region, the solution has oscillations that are, at most, as quickly oscillating as dictated by the total energy. Near the $r_{2}=0$ and $r_{3}=0$ edges, however, the basis manages to describe more rapidly oscillating waves.

We display two hyperangular cuts in Figs. 4 and 5. We recall that we use the same conventions as in $[25,35]$ for the hyperspherical coordinates, namely $\rho=\sqrt{r_{2}^{2}+r_{3}^{2}}$ and 

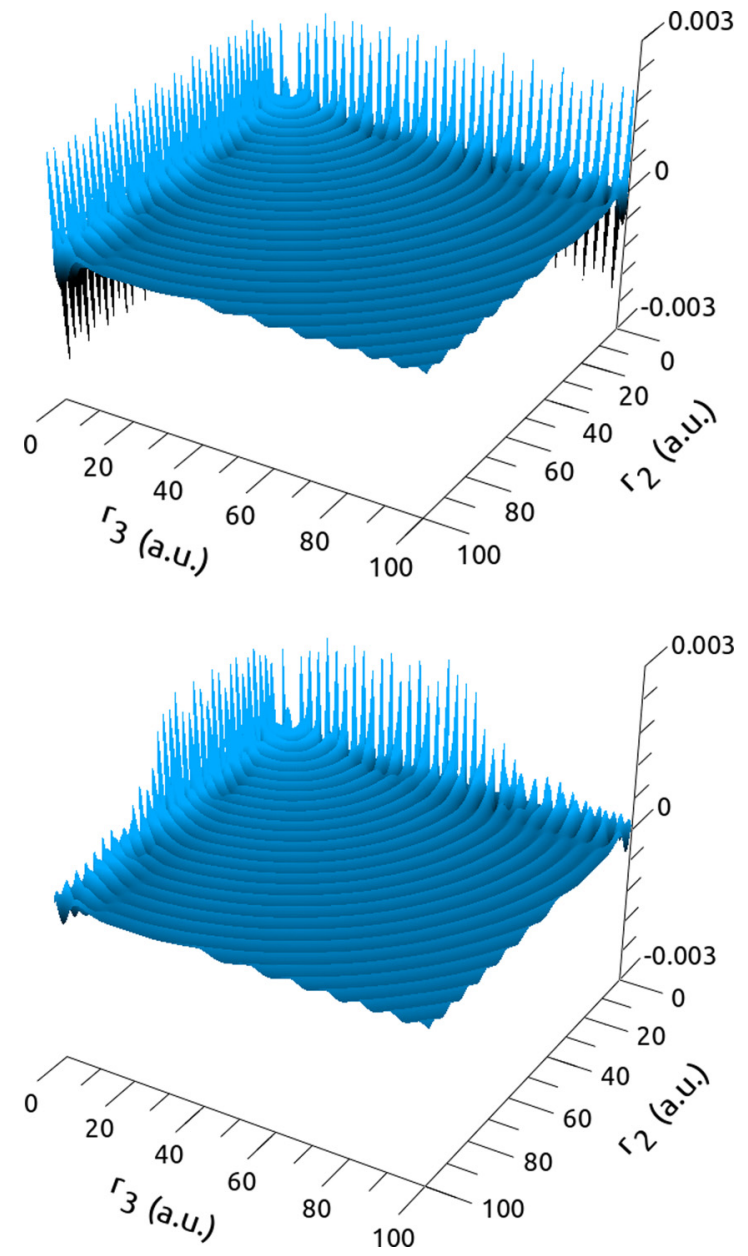

FIG. 2. (Color online) $(e, 3 e)$ scattering functions, real part, evaluated with (a) square-well-type Sturmian basis, (b) YukawaSturmian-type basis. The SW basis allows for a better characterization of what will be shown to be single-ionization channels, close to the $r_{2}=0$ and $r_{3}=0$ edges of both plots.

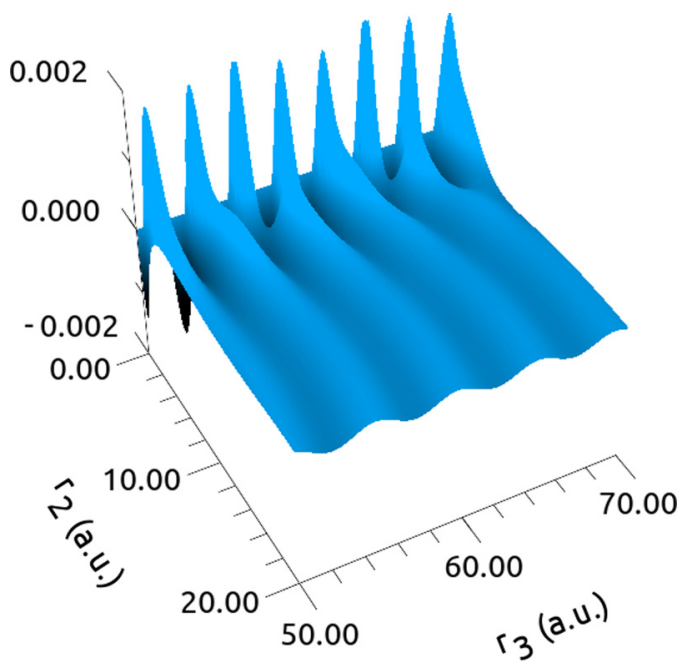

FIG. 3. (Color online) Closeup view of the structures arising near $r_{2}=0$. They clearly have a frequency different from that of the double continuum hyperspherical fronts.

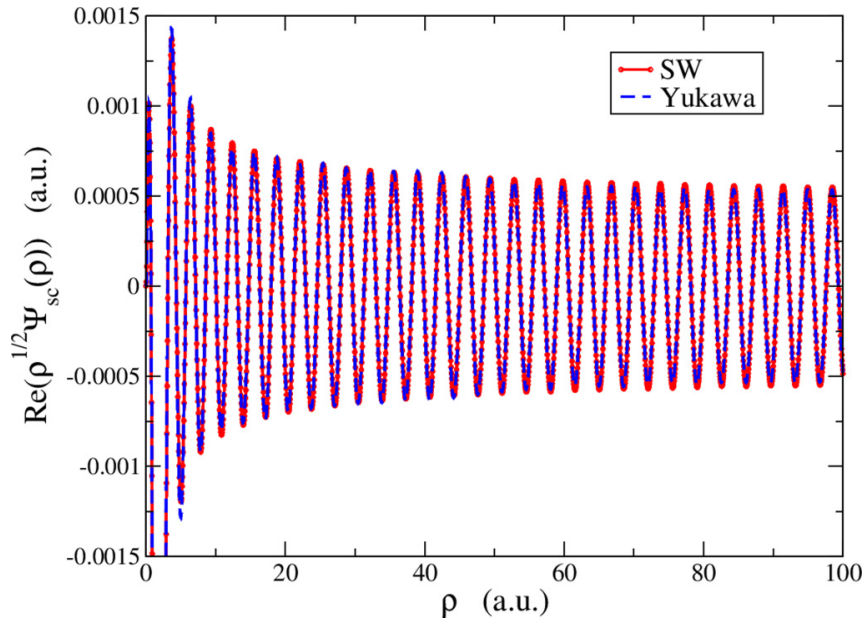

FIG. 4. (Color online) Hyperangular cut at $\alpha=\pi / 4$ of the $(e, 3 e)$ function calculated with the SW (solid line) and Yukawa (dashed line) sets. The agreement is excellent in the whole domain.

$\tan (\alpha)=\frac{r_{3}}{r_{2}}$. One of the three-body function hyperangular cuts is performed at $\alpha=45^{\circ}$ (Fig. 4) and the other one at $\alpha=15^{\circ}$ (Fig. 5). The agreement of both SW and Yukawa representations in the double continuum region is excellent. For a given number of basis elements the SW basis deals in a very effective way with the continuum three-body problem, better than the Yukawa basis.

Finally, there is an important aspect we have to stress regarding the Sturmian basis. The basis elements in (17) are uncorrelated, in the sense that no explicit interelectronic coordinate $r_{23}$ is present. They do not contain by themselves the double-ionization asymptotic behavior, i.e., the Peterkop hyperspherical wave front, and they do not include the $\frac{1}{\rho^{5 / 2}}$ decay. This means the basis actually is able to expand those structures very accurately. These results do not depend on the details of the generating potentials included in the calculation, SW or Yukawa interactions in the present contribution, and

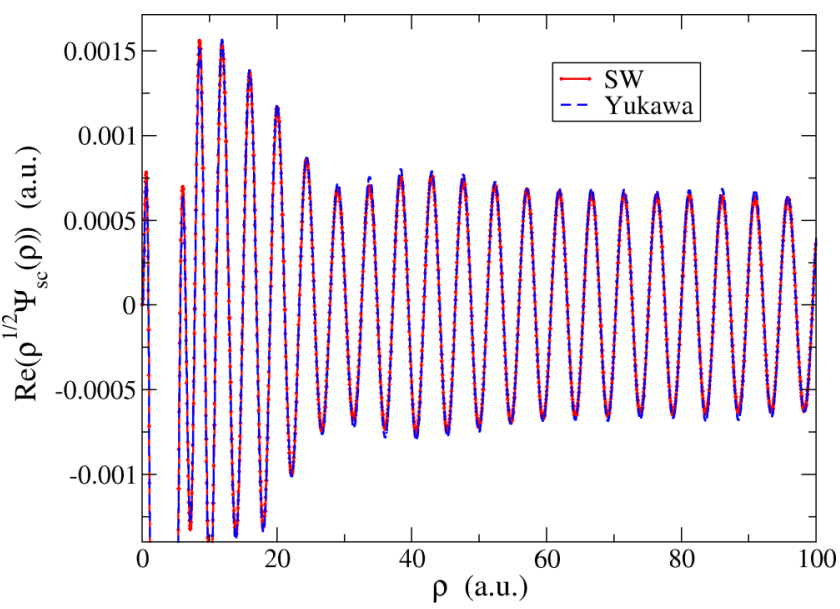

FIG. 5. (Color online) Hyperangular cuts along $\alpha=\pi / 12$ of the $(e, 3 e)$ function calculated with the $\mathrm{SW}$ (solid line) and Yukawa (dashed line) sets. The agreement is excellent in the whole domain. 
the wave function is equally accurate in the double-ionization regime.

\section{ISOLATING SINGLE-IONIZATION CHANNELS}

In this section we are going to analyze more deeply the solution obtained when using the SW generating potential basis. In particular, we will concentrate our attention on the part of the full function described by Eq. (12). We are going to effectively show that these quickly oscillating features observed on the edges, near the axes $r_{2}=0$ and $r_{3}=0$ in Figs. 2(a), 2(b), and 3, do not represent double continuum electrons.

Single-ionization channel three-body functions have one of the electrons bound to the atomic core, while the other electron is free. These states can be found on the full $(e, 3 e)$ function $\phi_{s c}^{+}\left(r_{2}, r_{3}\right)$, selecting only the parts which are the product of one $\mathrm{He}^{+}$bound state $\phi_{n}\left(r_{i}\right)(n=1,2, \ldots)$ times a continuum wave $\xi_{n}\left(r_{i}\right)$, for $i=2,3$. To extract these channels, one can project the full $\phi_{s c}^{+}\left(r_{2}, r_{3}\right)$ solution onto a given bound state $\phi_{n}$, obtaining each continuum $\xi_{n}$. Taking into account the symmetry of the states, each single-ionization channel $\tilde{\psi}_{n}\left(r_{2}, r_{3}\right)$ would take the following shape:

$$
\tilde{\psi}_{n}\left(r_{2}, r_{3}\right)=\xi_{n}\left(r_{2}\right) \phi_{n}\left(r_{3}\right)+\xi_{n}\left(r_{3}\right) \phi_{n}\left(r_{2}\right)-\phi_{n}\left(r_{2}\right) \phi_{n}\left(r_{3}\right),
$$

where the last term avoids the double counting of $\phi_{n}\left(r_{2}\right) \phi_{n}\left(r_{3}\right)$.

Figure 6(a) shows the resulting wave function obtained summing the first five single-ionization channels $\tilde{\psi}_{n}\left(r_{2}, r_{3}\right)$. It is clear that the single-ionization channels have a significant amplitude near the axis, and occupy a region in space which is complementary to the pure double-ionization channel, which is plotted in Fig. 6(b) without the first five $\tilde{\psi}_{n}\left(r_{2}, r_{3}\right)$ states. An important difference in magnitude between the single- and double-ionization channels can also be appreciated.

We now turn our attention to the functions $\xi_{n}\left(r_{i}\right)$ to show that they effectively satisfy the conditions expected for single-ionization channels. The $\xi_{n}\left(r_{2}\right)$ states should describe electron 2 leaving the $\mathrm{He}^{+}$with a specific energy and seeing an asymptotic unitary charge (due to screening from electron $3)$. The energy for the ejected electron has to be $E+\frac{4}{2 n^{2}}$.

To prove that the energy and asymptotic charge are the correct ones, we generated a new one-electron Sturmian basis set $\mathcal{S}_{m l}^{(n)}\left(r_{2}\right)$ with energy $E+\frac{4}{2 n^{2}}$ and core charge $Z=1$. The $n$ index labels the energy $E+\frac{4}{2 n^{2}}$ of this basis set, while $l$ angular momentum and $m$ enumerate the elements. We define the new auxiliary GSF basis, $\mathcal{S}_{m l}^{(n)}\left(r_{2}\right)$, using a generating potential such that they can be considered Coulomb wave functions with pure outgoing behavior for $r>70$ a.u.

We make use of this basis set to further expand each single continuum $\xi_{n}\left(r_{2}\right)$ state, and analyze the asymptotic and convergence properties. The $\xi_{n}\left(r_{2}\right)$ were evaluated in the same 140-a.u. box as the three-body wave functions they are obtained from. Thus, if the resulting re-expansion matches the ejected electron function in that region, one can conclude that the energy and asymptotic charge of $\xi_{n}\left(r_{2}\right)$ are those expected, i.e., $E+\frac{4}{2 n^{2}}$ and +1 , respectively. This, in turn, definitely will prove that the structures located near the axes are indeed single-ionization channels. (a)

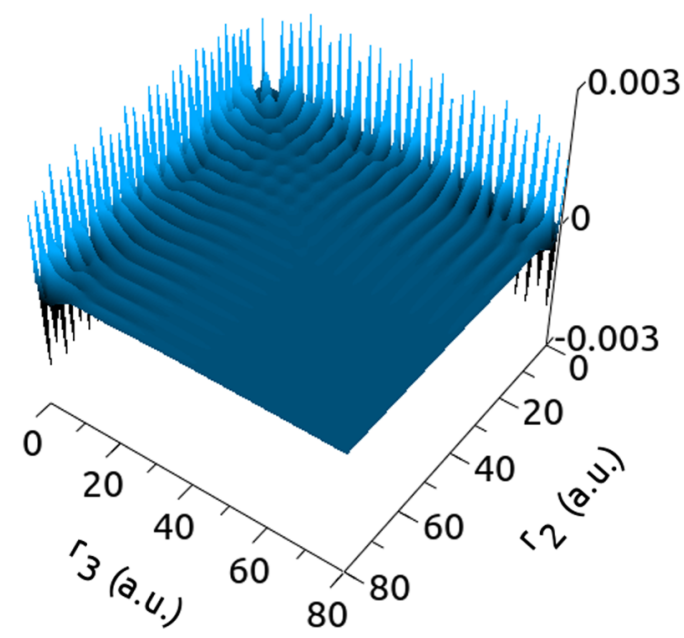

(b)

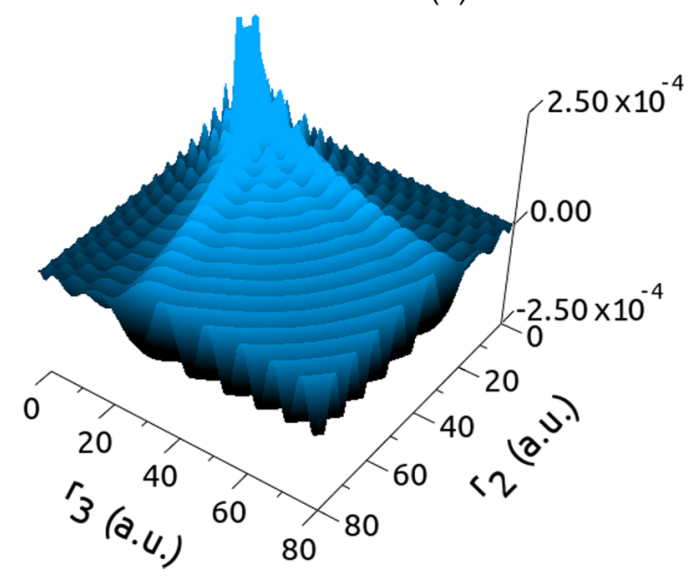

FIG. 6. (Color online) Full scattering function decomposed into single-ionization and double-ionization channels, comprising from $n=1$ to $n=5$ of the resulting $\mathrm{He}^{+}$ion.

In Fig. 7 we show an illustration of this procedure for $n=4$, and plot the function $\xi_{n}\left(r_{2}\right)$ compared to its expansion in the basis set $\mathcal{S}_{m l}^{(n)}\left(r_{2}\right)$. The agreement between these functions is excellent, proving that the ionized electron leaves the atom with the precise energy corresponding to the total energy plus the $\mathrm{He}^{+} n s$ bound electron energy, namely $\frac{4}{2 n^{2}}$. The choice of $Z=1$ as the effective charge for the ionic residual $\mathrm{He}^{+}$ leads to the optimal agreement between the expansion and the single-ionization channel obtained from the three-body function.

The expansion in this basis also shows that the asymptotic behavior for the first seven single-ionization channels is attained for distances smaller than 70 a.u., and in some cases, as small as 55 a.u. This implies that calculations for single-ionization channels do not require huge spatial domains in order to be sure that the asymptotic behavior has been reached, speeding up calculation time. These results held true for all the single-ionization channels studied here.

When the ionization or ionization excitation takes place, the projectile leaves a given amount of energy $E_{a}$ in the target 


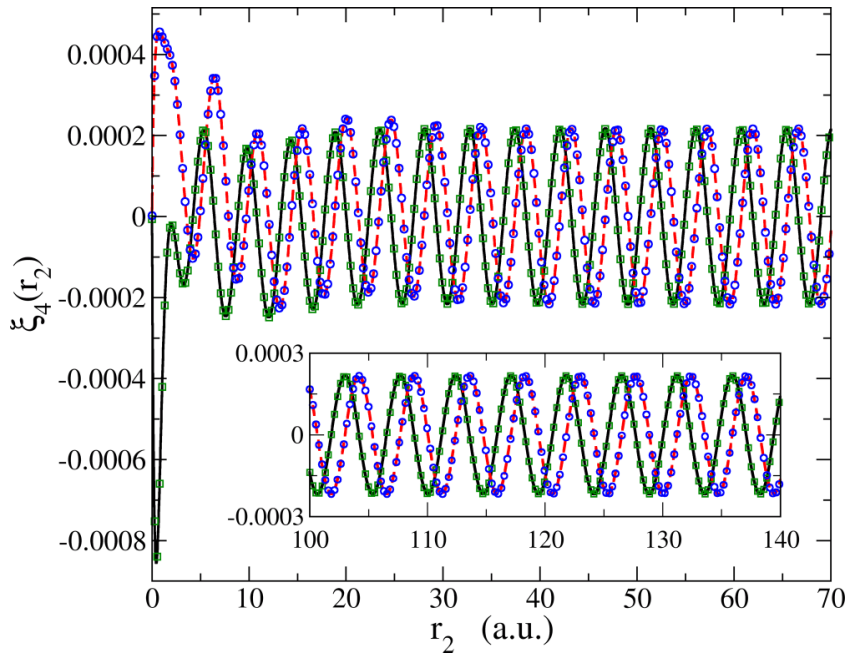

FIG. 7. (Color online) $\xi_{4}\left(r_{2}\right)$ and its corresponding Sturmian reexpansion. Real part of $\xi_{4}\left(r_{2}\right)$ in black continuous line, imaginary part of $\xi_{4}\left(r_{2}\right)$ in red dashed line. The real part of the re-expansion is plotted in green squares and the imaginary part in blue circles. The re-expansion basis has energy 0.8840 and charge -1 , and it is a basis within 70 a.u., i.e., it has no expanding power beyond. This shows that the asymptotic behavior of $\xi_{4}\left(r_{2}\right)$ is indeed the given by that complementary energy and asymptotic charge as evidenced in the inset.

subsystem. In all the processes that may occur the electrons share the energy received, such that $E_{a}=E_{2}+E_{3}$. Assuming, as done before, that $E_{a}>0$, then double ionization may occur and then $E_{2}>0$ and $E_{3}>0$. Ionization excitation is also an open channel implying that only one of the electrons is in the continuum, while the second one is bound. In this case, the energy of the ejected electron must be $E_{a}+1$ $E_{3} \mid=E_{2}$, where $E_{3}$ is the energy of the bound state. In the calculations performed to obtain the results for the double continuum we set the basis energy equal to the total one. Therefore, the basis energy differs in magnitude from the value corresponding to the single-ionization channels. Those singleionization channels matching or approximately matching the basis energy will be better represented by three-body basis, and no appreciable spurious contributions will be present in their asymptotic form. They adopt the correct, purely outgoing behavior. However, less excited states can make up for an ejection energy significantly larger than the total energy. This is shown in Fig. 8 where we compare $\xi_{2}\left(r_{2}\right)$ with its expansion in the basis set $\mathcal{S}_{m l}^{(2)}\left(r_{2}\right)$. There, we can see that $\xi_{2}\left(r_{2}\right)$ has slightly different real and imaginary amplitudes, and that the asymptotic behavior of $\xi_{2}\left(r_{2}\right)$ is not completely matched by

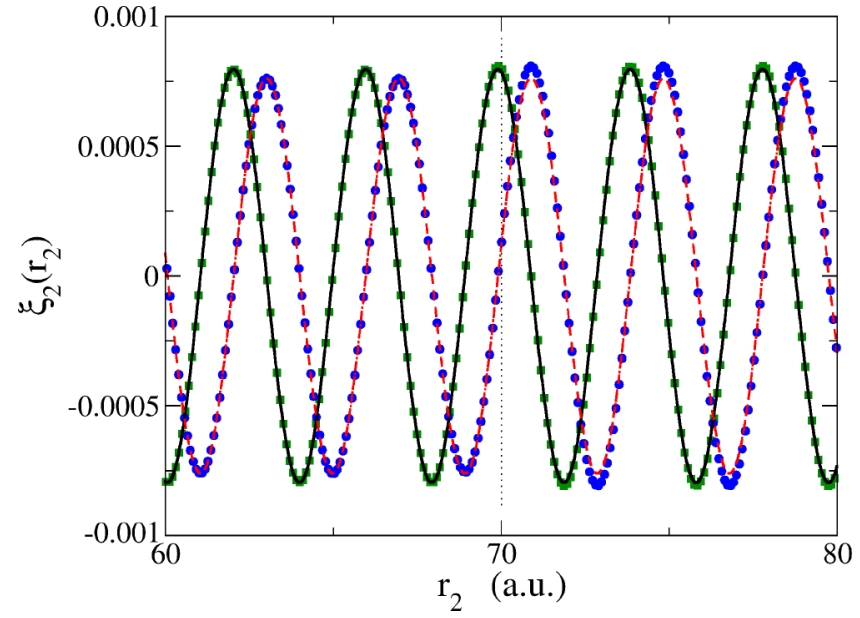

FIG. 8. (Color online) $\xi_{2}\left(r_{2}\right)$ and its corresponding Sturmian reexpansion. Real part of $\xi_{2}\left(r_{2}\right)$ in black continuous line, imaginary part of $\xi_{2}\left(r_{2}\right)$ in red dashed line. The real part of the re-expansion is plotted in green squares and the imaginary part in blue circles. The re-expansion basis has energy 1.2590 and charge -1 , and it is a basis within 70 a.u.; i.e., it has no expanding power beyond. In this figure we see, as opposed to Fig. 7, that three-body basis does not account properly for $\xi_{2}\left(r_{2}\right)$, as can be inferred from the fact that the real and imaginary parts of $\xi_{2}\left(r_{2}\right)$ do not have equal amplitudes.

the purely outgoing behavior of the basis $\mathcal{S}_{m l}^{(2)}\left(r_{2}\right)$. The same is observed for $\xi_{n}\left(r_{2}\right)$ (with $E=0.7590$ a.u.), and $n=1,2,3$. We can infer that this implies an asymptotic behavior consisting of an outgoing wave plus some small incoming contribution. The amount of incoming contribution diminishes when $n$ increases because the energy corresponding to the studied state tends to that of the $\left\{S_{n_{a}}^{+}\left(r_{2}\right), S_{n_{b}}^{+}\left(r_{3}\right)\right\}$ sets from (18).

To study the amount of unwanted incoming wave flux contained in the ejected electron functions $\xi_{n}\left(r_{2}\right)$, we express them in their asymptotic region as a combination of pure Coulomb incoming and outgoing functions:

$$
\xi_{n}(r) \rightarrow A_{+} H^{+}\left(Z, E_{n}, r\right)+A_{-} H^{-}\left(Z, E_{n}, r\right) .
$$

Evaluation of (21) at two different radii, $R_{1}, R_{2}$, within the asymptotic range of each $\xi_{n}(r)$ provides two equations, from which outgoing and incoming amplitudes can be obtained:

$$
\begin{aligned}
& A_{+} H^{+}\left(Z, E_{n}, R_{1}\right)+A_{-} H^{-}\left(Z, E_{n}, R_{1}\right)=\xi_{n}\left(R_{1}\right), \\
& A_{+} H^{+}\left(Z, E_{n}, R_{2}\right)+A_{-} H^{-}\left(Z, E_{n}, R_{2}\right)=\xi_{n}\left(R_{2}\right) .
\end{aligned}
$$

For $A_{+}, A_{-}$Eqs. (22) and (23) yield

$$
\begin{aligned}
& A_{+}=\frac{\xi_{n}\left(R_{1}\right) H^{-}\left(Z, E_{n}, R_{2}\right)-\xi_{n}\left(R_{2}\right) H^{-}\left(Z, E_{n}, R_{1}\right)}{H^{+}\left(Z, E_{n}, R_{1}\right) H^{-}\left(Z, E_{n}, R_{2}\right)-H^{-}\left(Z, E_{n}, R_{1}\right) H^{+}\left(Z, E_{n}, R_{2}\right)}, \\
& A_{-}=\frac{\xi_{n}\left(R_{2}\right) H^{+}\left(Z, E_{n}, R_{1}\right)-\xi_{n}\left(R_{1}\right) H^{+}\left(Z, E_{n}, R_{2}\right)}{H^{+}\left(Z, E_{n}, R_{1}\right) H^{-}\left(Z, E_{n}, R_{2}\right)-H^{-}\left(Z, E_{n}, R_{1}\right) H^{+}\left(Z, E_{n}, R_{2}\right)},
\end{aligned}
$$

with $H^{+}\left(Z, E_{n}, r\right), H^{-}\left(Z, E_{n}, r\right)$ denoting the outgoing or incoming Coulomb functions with charge $Z$ and energy
$E_{n}$ within our zero-angular-momentum example. If the $A_{-}$ amplitude is appreciably smaller than $A_{+}$, there is essentially 
TABLE I. Transition amplitudes for the single-ionization channels: absolute values of the outgoing wave components and quotients of the moduli of incoming to outgoing amplitudes, expressed as percentage. The quotients should ideally be zero, and we observed that if smaller than $\sim 5.0$ the asymptotic behavior of $\xi_{n}\left(r_{2}\right)$ is sufficiently close to a pure outgoing wave. We also show the single-ionization amplitudes for the first two channels calculated from separate three-body resolutions with basis energies $E_{s}$ equaling that of the ejected electron energy for a given channel. These separate resolutions required smaller box radii: 30 a.u. for $a, 60$ a.u. for $b$ and $c$; see text for details.

\begin{tabular}{cccc}
\hline \hline$n$ & Ejected electron energy (a.u.) & $10^{3}\left|A_{+}\right|$ & $\left|A_{-} / A_{+}\right|(\%)$ \\
\hline 1 & 2.759000 & 1.3524 & 10.6 \\
2 & 1.259000 & 0.7161 & 6.14 \\
3 & 0.981222 & 0.3345 & 3.30 \\
4 & 0.884000 & 0.2126 & 1.92 \\
5 & 0.839000 & 0.1521 & 1.21 \\
6 & 0.814555 & 0.1149 & 0.79 \\
7 & 0.799816 & 0.0912 & 0.55 \\
$a$ & 2.759000 & 1.3625 & 0.02 \\
$b$ & 2.759000 & 1.3426 & 0.15 \\
$c$ & 1.259000 & 0.4433 & 0.32 \\
\hline \hline
\end{tabular}

no incoming flux. Table I contains the outgoing amplitudes and the relative presence of incoming components. At first glance, the table shows smaller $\left|A_{-} / A_{+}\right|$quotients for more highly excited states. Ideally, the incoming amplitudes, and thus the quotients, should be zero. The approximate description of this channel is then observed as the presence of a nonzero incoming component. This is more pronounced when the bound electron stays in a low-lying state, as can be seen in the $\left|A_{-} / A_{+}\right|$values for the first few $(n=1,2)$ ones. The cause is that the ejection energies, 2.7590 and 1.2590 a.u., are not close enough to the basis energy one, 0.7590 a.u. We also compare three other cases. The first one, noted as $a$ in the table, corresponds to a separate three-body resolution where the basis energy was set to 2.7590 a.u. in order to have the first single-ionization channel well characterized. This can be clearly seen from the fact that $\left|A_{-} / A_{+}\right|$has now a small value compared to $n=1$. In this case, the whole solution was obtained in a box of just 30 a.u. The next two values in the table, labeled as $b$ and $c$, are solutions obtained in boxes of 60 a.u. for each coordinate, with energies 2.7590 a.u. and 1.2590 a.u. for $b$ and $c$, respectively. The case solved with $E_{s t}=E_{a}$ still provides a good description of $A_{+}$, as can be seen when comparing 1, 2, and 3 with $a, b$, and $c$.

If precise information concerning those single-ionization states is needed, separate resolutions with basis energies matching the ejection energy could be convenient. As we have shown, those deeply bound channels become asymptotic at around 50 a.u., and they needed radial domains which are considerably smaller than those for a double-ionization calculation. From those smaller domains the corresponding ionization amplitudes can be singled out as stated in this section. The separation of the different channels we performed here can be related to the work done by Randazzo and Ancarani [36]. There the authors explicitly mentioned that the ionization cross section should be extracted from the region of the configuration space where the single-ionization channel are excluded. In this paper we showed that those channels are present and that they are very significant near the borders.

There will still be a tiny amount of incoming amplitude, even when the energy of the given channel is very close to the basis energy, due to the fact that the basis set has, as asymptotic charge in the auxiliary potential $\mathcal{U}(r), Z=-2$, and the correct single-ionization solution should have $Z=-1$. The choice of $Z=-2$ has two advantages. First, it eliminates the electron-nucleus terms from the Hamiltonian. Second, in conjunction with the SW basis, it provides the basis elements which are indistinguishable from the bound $\mathrm{He}^{+}$states, which in turn simplify the extraction of each $\xi_{n}\left(r_{i}\right)$.

The present discussion accounts for all the details at the wave-function level. However, calculation of cross sections involves integrals of the wave function. We have shown in the present $(e, 3 e)$ approximation [25] that convergence is obtained at the cross-section level with the same GSF basis as that presented here. Therefore, contributions of incoming flux are small and do not modify the cross sections in the energy regime studied here.

\section{SUMMARY AND OUTLOOK}

We have presented a study of the details of the channels involved in the $\left(e^{-}, \mathrm{He}\right)$ collision, within a Temkin-Poet approximation. We provided a brief recapitulation of the properties of the generalized Sturmian functions method. Since there is a wide range of generating potentials that can be used to shape the basis set, we described the details of two main sets (square-well and Yukawa potentials) that have been used by our group and their role when solving three-body problems. Both basis sets are complete, and we have shown that they are completely equivalent to solve the double-ionization continuum. Better results were obtained near the edges of the domain with a basis generated with a square-well potential. That basis has its expanding power more evenly spread though the radial interval, as opposed to the Yukawa potential, that concentrates the basis near the origin. Besides, it contains elements that are numerically indistinguishable from the auxiliary potential bound states. These turned out to be useful whenever the goal is the analysis of the the single-ionization channels. A way to isolate the single-ionization functions was outlined, involving the projection onto $\mathrm{He}^{+}$states. In particular, the usage of the square-well-type basis made these projections unnecessary since it contains states that are numerically indistinguishable from those of the $\mathrm{He}^{+}$core. Functions corresponding to an ejected electron from the single-ionization components were clearly shown to be included in the full accurate three-body solution. They have an energy which is complementary to the newly bound $\mathrm{He}^{+}$electron, and correspond to an asymptotic unitary charge due to screening. This kind of analysis has been possible thanks to one of the main features of the GSF: the basis energy enters the calculation as a parameter, and can be changed according to the details of the physics under investigation. These results show the capabilities of the Sturmian functions method to deal with the single- and double-ionization channels. 
Our group has also presented a hyperspherical formulation, in terms of $(\rho, \alpha)$ instead of $\left(r_{2}, r_{3}\right)$, for the GSF method $[25,35,37]$. We are currently working to establish the suitabilities of both the spherical and hyperspherical formulations. The hyperspherical version has the correct Peterkop conditions built in, and thus is unparalleled in the double continuum description. However, the discrete channels will pose two challenges to that formulation. The first one refers to the various energies of the single-ionization channels, and can be addressed in the same way as with the spherical formulation, albeit in this case the projection onto a single electron $\mathrm{He}^{+}$(depending only on $r_{2}$ or $r_{3}$ ) state is notoriously more convoluted. This is due to the fact that the projection onto a $\mathrm{He}^{+}$state consist of separable one-dimensional (1D) integrals within the spherical formulation, but necessarily $2 \mathrm{D}$ integrals within the hyperspherical one. The second difficulty is related to the $\frac{1}{\rho^{5 / 2}}$ decay of the hyperradial basis elements, which does not match the nondecaying amplitude of the single-ionization channels. Up to this date, both Sturmian formulations of scattering problems are useful [25] and, in the light of this section, complementary to provide physical insight of collision problems.

Two different approaches are being explored for further improving the methodology. The first one is the utilization, on the same calculations, of basis sets $\Xi_{n_{a}, n_{b}}\left(r_{2}, r_{3}\right)$ with different energies to account for the first few single-ionization channels that are less well characterized by the basis elements with energy equaling $E$. Also, some bound basis elements should be included to characterize the bound states of the singly ionized atomic core. For this there is the option of employing combinations with $\phi_{n}\left(r_{i}\right)$ times continuum elements. The $\phi_{n}\left(r_{i}\right)$ can even be replaced by elements stemming from a negative energy basis set altogether.

The second, more ambitious scheme, consists of a hybrid utilization of hyperspherical and spherical basis sets, in the same spirit as the mixed proposal of Ref. [38]. The double continuum should be properly dealt with by the hyperspherical formulation, while for the single continuum parts better performance is expected from the spherical one.

Finally, one could study a mixed-type basis, summing up the Yukawa and square well to make a new generating potential. The resulting Sturmian set would be a middle ground between the two cases considered in this contribution: It would have a fair concentration of oscillations near the origin, but with the expanding power reaching the maximum radius. These types of potentials are being studied and will be used in future publications.

\section{ACKNOWLEDGMENTS}

We would like to acknowledge the support of projects ANPCyT (PICT08/0934) (Argentina) and PIP 200901/552 CONICET (Argentina). We also acknowledge the support by PGI (24/F059) of the Universidad Nacional del Sur and project C6/348 from Universidad Nacional de Cuyo.
[1] L. U. Ancarani, C. Dal Cappello, and G. Gasaneo, J. Phys.: Conf. Ser. 212, 012025 (2010).

[2] A. Lahmam-Bennani, I. Taouil, A. Duguet, M. Lecas, L. Avaldi, and J. Berakdar, Phys. Rev. A 59, 3548 (1999).

[3] I. Taouil, A. Lahmam-Bennani, A. Duguet, and L. Avaldi, Phys. Rev. Lett. 81, 4600 (1998).

[4] C. W. McCurdy, M. Baertschy, and T. M. Rescigno, J. Phys. B 37, R137 (2004).

[5] I. Bray and A. T. Stelbovics, Phys. Rev. A 46, 6995 (1992).

[6] A. Kheifets, I. Bray, A. Lahmam-Bennani, A. Duguet, and I. Taouil, J. Phys. B 32, 5047 (1999).

[7] A. D. Alhaidari, E. J. Heller, H. A. Yamani, and M. H. Abdelmonem, The J-matrix method-Developments and application (Springer, Dordretch, Netherlands, 2008).

[8] V. A. Knyr, V. V. Nasyrov, and Yu. V. Popov, Correlation and Polarization in Photonic, Electronic, and Atomic Collisions, AIP Conf. Proc. No. 697, edited by G. F. Hanne et al. (AIP, Melville, NY, 2003), p. 76.

[9] S. A. Zaytsev, V. A. Knyr, and Yu. V. Popov, Phys. At. Nucl. 70, 676 (2007).

[10] Z. Papp and C. Y. Hu, Phys. Rev. A 66, 052714 (2002).

[11] M. S. Pindzola, Sh. A. Abdel-Naby, J. Colgan, and A. Dorn, J. Phys. B 45, 215208 (2012).

[12] M. S. Pindzola and F. J. Robicheaux, Phys. Rev. A 61, 052707 (2000).

[13] D. A. Horner, C. W. McCurdy, and T. N. Rescigno, Phys. Rev. A 71, 012701 (2005).

[14] V. V. Serov, V. L. Derbov, B. B. Joulakian, and S. I. Vinitsky, Phys. Rev. A 75, 012715 (2007).
[15] A. L. Frapiccini, V. Y. Gonzalez, J. M. Randazzo, F. D. Colavecchia, and G. Gasaneo, Int. J. Quantum Chem. 107, 832 (2007).

[16] M. J. Ambrosio, J. A. Del Punta, K. V. Rodriguez, G. Gasaneo, and L. U. Ancarani, J. Phys. A 45, 015201 (2012).

[17] A. L. Frapiccini, J. M. Randazzo, G. Gasaneo, and F. D. Colavecchia, Phys. Rev. A 82, 042503 (2010).

[18] A. L. Frapiccini, G. Gasaneo, F. D. Colavecchia, and D. Mitnik, J. Electron Spectrosc. Relat. Phenom. 161, 199 (2007).

[19] J. M. Randazzo, L. U. Ancarani, G. Gasaneo, A. L. Frapiccini, and F. D. Colavecchia, Phys. Rev. A 81, 042520 (2010).

[20] J. M. Randazzo, A. L. Frapiccini, F. D. Colavecchia, and G. Gasaneo, Phys. Rev. A 79, 022507 (2009)

[21] D. M. Mitnik, J. M. Randazzo, and G. Gasaneo, Phys. Rev. A 78, 062501 (2008).

[22] G. Gasaneo, L. U. Ancarani, D. M. Mitnik, J. M. Randazzo, A. L. Frapiccini, and F. D. Colavecchia, Adv. Quantum Chem. 67, 153 (2013).

[23] J. M. Randazzo, F. Buezas, A. L. Frapiccini, F. D. Colavecchia, and G. Gasaneo, Phys. Rev. A 84, 052715 (2011).

[24] A. L. Frapiccini, J. M. Randazzo, G. Gasaneo, and F. D. Colavecchia, J. Phys. B 43, 101001 (2010).

[25] G. Gasaneo, D. M. Mitnik, J. M. Randazzo, L. U. Ancarani, and F. D. Colavecchia, Phys. Rev. A 87, 042707 (2013).

[26] C. J. Joachain, Quantum Collision Theory (North-Holland, Amsterdam, 1983), Chap. 6.

[27] O. Gosckinski, Preliminary research report no. 217, Quantum Chemistry Group, Uppsala University, 1968 (originally unpublished). Included as an Appendix in Adv. Quantum Chem. 41, 51 (2003). 
[28] J. Avery and J. Avery, Generalized Sturmians and Atomic Spectra (World Scientific, Singapore, 2006).

[29] J. M. Randazzo, A. L. Frapiccini, F. D. Colavecchia, and G. Gasaneo, Int. J. Quantum Chem. 109, 125 (2009).

[30] E. O. Alt and A. M. Mukhamedzhanov, Phys. Rev. A 47, 2004 (1993).

[31] R. Peterkop, Theory of Ionization of Atoms by Electron Impact (Colorado Associated University Press, Boulder, 1977).

[32] A. S. Kadyrov, A. M. Mukhamedzhanov, A. T. Stelbovics, I. Bray, and F. Pirlepesov, Phys. Rev. A 68, 022703 (2003).

[33] A. S. Kadyrov, I. Bray, A. M. Mukhamedzhanov, and A. T. Stelbovics, Ann. Phys. 324, 1516 (2009).
[34] P. A. Macri, J. E. Miraglia, C. R. Garibotti, F. D. Colavecchia, and G. Gasaneo, Phys. Rev. A 55, 3518 (1997).

[35] G. Gasaneo, D. M. Mitnik, A. L. Frapiccini, F. D. Colavecchia, and J. M. Randazzo, J. Phys. Chem. A 113, 14573 (2009).

[36] L. U. Ancarani and J. M. Randazzo, J. At. Mol. Sci. 4, 193 (2013).

[37] D. M. Mitnik, G. Gasaneo, and L. U. Ancarani, J. Phys. B 46, 015202 (2013).

[38] F. D. Colavecchia, F. Mrugala, G. A. Parker, and R. T. Pack, J. Chem. Phys. 118, 10387 (2003). 